\title{
Wi-Fi Coexistence with Duty Cycled LTE-U
}

\author{
Yimin Pang, ${ }^{1}$ Alireza Babaei, ${ }^{2}$ Jennifer Andreoli-Fang, ${ }^{3}$ and Belal Hamzeh ${ }^{3}$ \\ ${ }^{1}$ Department of Electrical, Computer and Energy Engineering, University of Colorado Boulder, Boulder, CO 80309, USA \\ ${ }^{2}$ Ofinno Technologies, Herndon, VA, USA \\ ${ }^{3}$ Cable Television Laboratories, Louisville, CO, USA
}

Correspondence should be addressed to Yimin Pang; yipa5803@colorado.edu

Received 1 July 2016; Revised 17 September 2016; Accepted 18 October 2016; Published 15 January 2017

Academic Editor: Adlen Ksentini

Copyright (C) 2017 Yimin Pang et al. This is an open access article distributed under the Creative Commons Attribution License, which permits unrestricted use, distribution, and reproduction in any medium, provided the original work is properly cited.

\begin{abstract}
Coexistence of Wi-Fi and LTE-Unlicensed (LTE-U) technologies has drawn significant concern in industry. In this paper, we investigate the Wi-Fi performance in the presence of duty cycle based LTE-U transmission on the same channel. More specifically, one LTE-U cell and one Wi-Fi basic service set (BSS) coexist by allowing LTE-U devices to transmit their signals only in predetermined duty cycles. Wi-Fi stations, on the other hand, simply contend the shared channel using the distributed coordination function (DCF) protocol without cooperation with the LTE-U system or prior knowledge about the duty cycle period or duty cycle of LTE-U transmission. We define the fairness of the above scheme as the difference between Wi-Fi performance loss ratio (considering a defined reference performance) and the LTE-U duty cycle (or function of LTE-U duty cycle). Depending on the interference to noise ratio (INR) being above or below $-62 \mathrm{dbm}$, we classify the LTE-U interference as strong or weak and establish mathematical models accordingly. The average throughput and average service time of Wi-Fi are both formulated as functions of Wi-Fi and LTE-U system parameters using probability theory. Lastly, we use the Monte Carlo analysis to demonstrate the fairness of Wi-Fi and LTE-U air time sharing.
\end{abstract}

\section{Introduction}

The rapidly growing demand of wireless network services has led the mobile network operators (MNOs) to look into the possibility of exploring unlicensed spectrum to offload the data traffic from the licensed bands. Most recently, $3 \mathrm{GPP}$ and other industry alliances are considering extending LTE into the unlicensed spectrum (3GPP has completed standardization of license assisted access (LAA) in release 13 (DL only) and enhanced LAA (eLAA) standardization, where UL access to unlicensed spectrum is also considered and is currently ongoing in 3PP release 14. LAA and eLAA incorporate listen-before-talk (LBT) for their channel access to the unlicensed spectrum. In addition, the LTE-U forum, an industry alliance formed by some vendors and mobile operators, has released an LTE supplemental downlink (SDL) coexistence specification, where an adaptive duty cycle based coexistence scheme is introduced. The term "LTE-U" is used instead of "LAA" in the LTE-U forum specification. Since this paper focuses on duty cycle based LTE on unlicensed spectrum, we use the short term LTE-U for convenience. However, the channel access approach introduced in this paper is not completely aligned with the channel access method introduced by the LTE-U forum specification due to nonadaptive duty cycle), to offload part of the LTE data traffic onto the unlicensed spectrum. Compared to data offload using Wi-Fi, this approach has the advantage of seamless integration into the existing LTE evolved packet core (EPC) architecture. In a proposal outlined in [1], three LTE-U modes are introduced as supplement downlink, TD-LTE-U carrier aggregation, and standalone, the first two of which were proposed to $3 \mathrm{GPP}$ as possible candidates.

Coexistence of heterogeneous networks such as Wi-Fi and LTE-U on the same band and their uncoordinated operations can potentially cause significant interference, degrading the performance of both systems. Solutions to manage the interference between such systems are therefore necessary for their successful coexistence. One straightforward method is to split the common radio channel through air time sharing between the Wi-Fi and LTE-U subsystems. With 


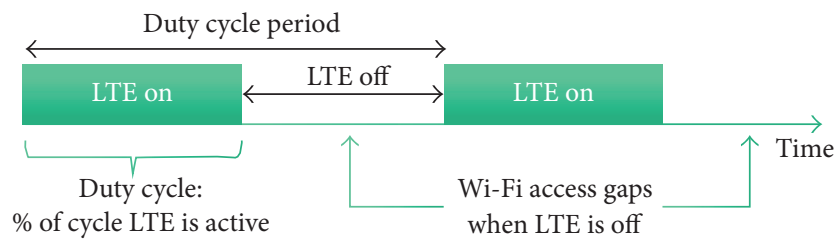

Figure 1: Air time sharing LTE-U.

this approach, LTE-U operates over the shared channel periodically (more specifically, the radio resources of LTE-U that reside in the unlicensed spectrum and are shared with Wi-Fi are utilized periodically), and during each period (the so-called duty cycle period, denoted as $T$ ), only a portion (defined by the LTE-U duty cycle $\alpha$ ) of the time is utilized for the LTE-U transmission, as shown in Figure 1. In this scheme, Wi-Fi has no cooperation with LTE-U. Wi-Fi stations have neither knowledge about the time length of duty cycle period nor the duty cycle; they simply access the shared channel by standard channel sensing and random back-off mechanisms.

In an LTE system, each user equipment (UE) communicates with a base station (eNB) in a deterministic manner through a centralized channel access control mechanism. The access time and OFDM subcarriers of an LTE frame are predetermined at $\mathrm{eNB}$, where the MAC scheduler considers the radio measurement and quality of service needed for each UE in its scheduling decisions. Given the above time sharing scheme and the LTE's centralized access structure, computation of the LTE-U performance in terms of throughput and service time is relatively simple and straightforward. However, the medium access mechanism used by Wi-Fi, controlled by distributed coordination function (DCF) protocol defined in IEEE 802.11 standard, is random and distributed. Therefore, we focus on the impact of LTE-U interference on Wi-Fi performance in this paper.

1.1. Previous Works. LTE-U and Wi-Fi coexistence are a relatively new area of research. Previous works in this area are summarized as follows: in [2], Wi-Fi and LTE-U coexistence in single floor and multiple floors environment at various densities are simulated. The results show that, without any interference management scheme, LTE-U system performance is slightly affected from $\mathrm{Wi}-\mathrm{Fi}$, whereas $\mathrm{Wi}-\mathrm{Fi}$ is significantly impacted by the LTE-U transmissions. This result is reinforced in [3] by computing the Wi-Fi successful channel accessing probability in the presence of LTE-U transmission. However, in [1], LTE-U is described as a better neighbor to $\mathrm{Wi}-\mathrm{Fi}$ than $\mathrm{Wi}-\mathrm{Fi}$ to itself as long as a proper coexistence mechanism (called CSAT) is applied. Authors in [4] present simulation results on spectrum efficiency comparison between Wi-Fi and LTE-U in a sparse deployment scenario. The paper, however, lacks sufficient details on the coexistence features and their effectiveness. Cano and Leith [5] proposed a duty cycle mechanism for LTE-U, which, by selecting an appropriate probability to access the channel and transmission duration, ensures proportional fairness among LTE-U and Wi-Fi nodes. Specifications regarding duty cycled based LTE-U are released and maintained by
LTE-U forum [6], where CSAT is officially introduced as the access mechanism.

On the other hand, the 3GPP study item technical report document [7] has listed listen-before-talk (LBT) as the required function for clear channel assessment for LTE LAA. The application of LBT may potentially enhance the coexistence behavior of Wi-Fi and LTE. Some analysis and performance test have been reported in [8-10].

1.2. Main Results. In this paper, we define Wi-Fi average saturation throughput $\mathscr{R}(T, \alpha, \mathscr{H})$ (in terms of bits/Wi-Fi slot time) and average service time $\mathscr{D}(T, \alpha, \mathscr{H})$ (in terms of Wi-Fi time slots) to be functions of $T, \alpha$, and $\mathscr{H}=\{q, L, n\}$, where the set $\mathscr{H}=\{q, L, n\}$ represents $n$-clients Wi-Fi subsystem with LTE-U to Wi-Fi collision probability (Wi-Fi transmission failure probability due to LTE-U transmission) $q$ and WiFi data payload length $L$ (the length of MAC data payload, in terms of bytes). Given $\mathscr{H}$, the throughput fairness (cf. Definition 2) of $(T, \alpha)$ air time sharing scheme is measured by the difference between average Wi-Fi saturation throughput loss ratio (with respect to the corresponding non-LTE-U duty cycle scenario $(\infty, 0, \mathscr{H})$ performance) and LTE-U duty cycle $\alpha$, that is, $(\mathscr{R}(\infty, 0, \mathscr{H})-\mathscr{R}(T, \alpha, \mathscr{H})) / \mathscr{R}(\infty, 0, \mathscr{H})-\alpha$. In a similar way, the average service time fairness is defined as $(\mathscr{D}(T, \alpha, \mathscr{H})-\mathscr{D}(\infty, 0, \mathscr{H})) / \mathscr{D}(\infty, 0, \mathscr{H})-\alpha /(1-\alpha)$. These two fairness measures indicate whether Wi-Fi will lose less or more than $\alpha$ portion of its performance (in the absence of LTE- $\mathrm{U}$ transmission) if $\alpha$ portion of the channel resource is shared with LTE-U.

Our first step is to analytically formulate $\mathscr{R}(T, \alpha, \mathscr{H})$ and $\mathscr{D}(T, \alpha, \mathscr{H})$ using a probabilistic framework. The following two key techniques are employed.

1.2.1. Only One Labeled Client Station among the $n$ WiFi Stations Being Affected by LTE-U Interference. As first introduced by [11], Wi-Fi DCF can be formulated into a Markov chain model, which was generalized later in $[12,13]$. But when LTE-U is considered, the Markov property no longer holds, because of the fact that when LTE-U is off, the $\mathrm{Wi}-\mathrm{Fi}$ transmission failure probability is only $\mathrm{Wi}-\mathrm{Fi}$ to Wi-Fi collision probability; when LTE-U is on, the Wi-Fi transmission failure probability depends on both Wi-Fi to Wi-Fi and LTE-U to Wi-Fi collision probability. For this issue, we make an assumption that only one client station among the $n$ stations, labeled as Sta-A, is affected by the LTE-U interference. The other $n-1$ stations render the Wi-Fi background traffic for the labeled station. When $n$ is chosen to be large enough, the background traffic could still be approximately modeled using the existing framework in [11-13]. Under this assumption, functions $\mathscr{R}(T, \alpha, \mathscr{H})$ and $\mathscr{D}(T, \alpha, \mathscr{H})$ are with respect to Sta-A.

1.2.2. Different Interference Levels Lead to Different Formulations. The Wi-Fi DCF employs CSMA/CA with binary exponential back-off algorithm. Depending on the energy level being detected, the back-off timer may or may not be frozen. In short, an LTE-U transmission with interference to noise ratio (INR) greater than $-62 \mathrm{dbm}$ or a neighbor Wi-Fi station transmission with INR greater than $-82 \mathrm{dbm}$ 
will cause the interfered Wi-Fi station to freeze its back-off timer. We refer to weak interference as the LTE-U interference with its INR being less than $-62 \mathrm{dbm}$ and strong interference as interference with INR greater than $-62 \mathrm{dbm}$. The mathematical formulations as well as the performance results are quite different between the cases of weak and strong LTE-U interference.

Other assumptions are just inherited from the existing framework by [11-13] on Wi-Fi DCF: (1) a transmission from one Wi-Fi station can be heard by all the other $n-1 \mathrm{Wi}$ Fi stations, and Wi-Fi to Wi-Fi INR is always greater than $-82 \mathrm{dbm}$; (2) collisions among Wi-Fi or LTE-U to Wi-Fi are the only causes to Wi-Fi failure transmission.

Then, we analyze the performance as well as the fairness numerically. Both the analytical functions built for the weak and strong LTE- $\mathrm{U}$ interference are computationally inefficient and characterizing the two performance functions in closed form is hard. On the other hand, implementing Monte Carlo analysis based on these two functions is simple. It is also difficult and meaningless to show the fairness over all possible combinations of $T, \alpha$, and $\mathscr{H}$. We focus our attention on the cases when LTE-U to Wi-Fi collision probability $q=1$, which has wide measure over real systems where LTE-U INR and Wi-Fi SNR are comparable. Other parameters are also selected in a reasonable range according to practical system setting. The results demonstrated in Section 4 support the conclusions below:

(1) Fix $q=1$ : under strong interference, air time sharing scheme could approximately achieve the fairness for some $(T, \alpha)$; under weak interference, air time sharing scheme is generally unfair.

(2) The fairness measure degrades almost linearly when LTE-U to Wi-Fi collision probability $q$ increases.

(3) The fairness measure degrades almost linearly when Wi-Fi payload length $L$ increases.

The rest of the paper is organized as follows: Section 2 presents the Wi-Fi and LTE-U coexistence model and formulates the problem; Section 3 characterizes the average WiFi saturation throughput and average service time in the presence of LTE-U duty cycle; the impact of duty cycled LTE$\mathrm{U}$ interference to Wi-Fi is discussed in Section 4; Section 5 concludes the paper.

\section{System Model and Problem Formulation}

In this section, we first introduce the generalized Markov chain model for Wi-Fi DCF, then we formulate the Wi-Fi and duty cycled LTE-U coexistence, and lastly we define the fairness measure.

2.1. Generalized Markov Chain Model for Wi-Fi DCF. In [11], the Wi-Fi DCF is formulated into a two-dimensional Markov chain; the $i$ th floor in the Markov chain (refer to Figure 2) stands for the random back-off process before the $i$ th transmission attempt, where $0 \leq i \leq M$, with contention window size $\mathrm{CW}_{i}=2^{i} \mathrm{CW}_{0}$, where $\mathrm{CW}_{0}$ is the contention window size of the 0th back-off. This Markov chain has transition probability $p\left(i_{n+1}, j_{n+1} \mid i_{n}, j_{n}\right.$ ) (with a slight abuse of notation, we temporarily use $n$ to denote the state at $n$th discrete moment),

$$
\begin{cases}1 & i_{n+1}=i_{n} ; j_{n+1}=j_{n}-1, \\ & j_{n} \neq 0, \\ 1-p_{i_{n}} & i_{n+1}=0, i_{n} \neq M-1 ; \\ & j_{n+1} \in\left\{0, \ldots, \mathrm{CW}_{0}\right\}, j_{n}=0, \\ \frac{p_{i_{n}}}{2^{i_{n+1}} \mathrm{CW}_{0}} & i_{n+1}=i_{n}+1, i_{n+1} \neq M-1 ; \\ & j_{n+1} \in\left\{0, \ldots, 2^{i_{n+1}} \mathrm{CW}_{0}\right\}, j_{n}=0, \\ 1 & i_{n+1}=0, i_{n}=M-1 ; \\ & j_{n+1} \in\left\{0, \ldots, \mathrm{CW}_{0}\right\}, j_{n}=0 .\end{cases}
$$

Let $\sigma$ be the duration of the Wi-Fi system slot time as defined in IEEE 802.11 standard. Throughout the paper, we normalize all the time variables to $\sigma$, which means $1 \mathrm{~s}$ is normalized to $1 / \sigma$. During each $(i, 0)$ state, a Wi-Fi station senses the channel; with probability $p_{i}$ it detects clear channel and transmits (or retransmits) a packet. If successful, the station stays idle or goes back to 0th contention level for a new packet; otherwise the failed packet will be retransmitted until it reaches the maximum number of retry attempts $M$. Before the $i$ th attempt, a random number is generated according to the uniform distribution $\operatorname{Unif}\left(0, \mathrm{CW}_{i}-1\right)$ and loaded into the back-off timer. The timer decreases the registered value by one per slot time, and once the back-off timer is reset, the station senses the channel for the $i$ th attempt.

Recall that a Wi-Fi station receiving Wi-Fi interference over $-82 \mathrm{dbm}$ will freeze its back-off timer; therefore the transition time between two neighbor states in the Markov chain may be more than one Wi-Fi system slot time. In order to use this Markov chain model to analyze the Wi-Fi service time, the Bianchi model in [11] is further generalized by $[12,13]$ after incorporating the following two further assumptions:

(1) The transition time $T_{d}$ between any two neighbor states (we call this unit decrement time for short, normalized to system slot time) is identically and independently distributed, and interference between any two Wi-Fi stations is above $-82 \mathrm{dbm}$ threshold.

(2) Based on assumption (1) and applying central limit theorem, the time interval from a back-off timer loads with an initial number $J_{i}$ before $i$ th attempt to the timer being reset is a Gaussian random variable with mean $J_{i} E\left(T_{d}\right)$.

We adopt the generalized Markov model of Wi-Fi in this paper.

Next we describe the failure probability $p_{i}$ in each retransmission trial. Let $\lambda$ be the probability that there is no packet ready to transmit, and let $\tau$ be the probability that a WiFi station transmits (or retransmits) a packet in a randomly chosen time slot given a packet just left the buffer and is ready to be transmitted. The number $\tau$ is a function of the number 


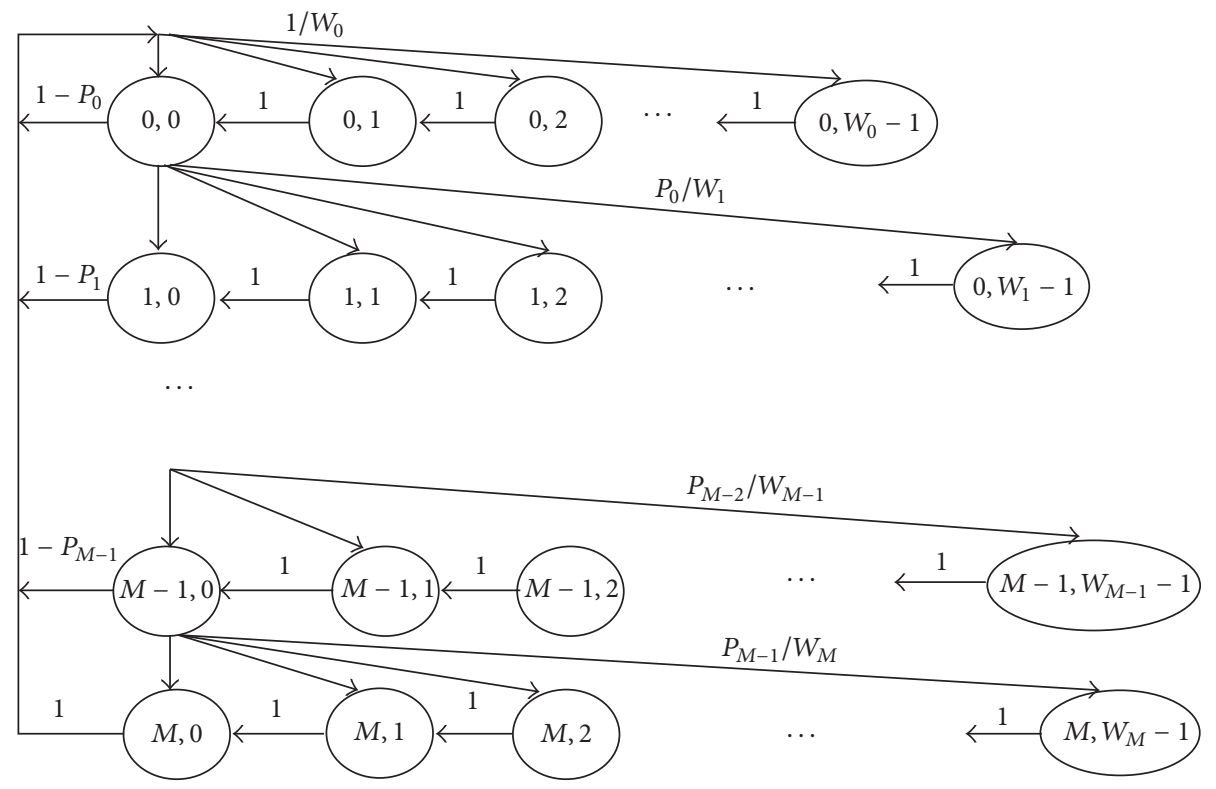

Figure 2: Markov model for Wi-Fi DCF (refer to [11]).

of Wi-Fi stations $n$ and $p_{i}$. Without LTE-U interference, the probability $p_{i}$ is simply the collision probability $p_{c}$ that at least two Wi-Fi stations transmit simultaneously, which is

$$
p_{i}=p_{c}=1-[1-(1-\lambda) \tau]^{n-1} .
$$

On the other hand, we have

$$
\tau=\sum_{i=0}^{M-1}\left(1-p_{c}\right) p(i, 0)+\left(1-p_{c}\right) p(M, 0)
$$

according to the transition probability defined in (1), where $p(i, j)$ is the stationary distribution of the Markov chain. There is no close form expression of the solution to $p_{i}$ and $\tau$, but given the system parameters and number of stations, they can be numerically computed. When the Wi-Fi system is saturated, the buffer in each station is never empty; that is, $\lambda=0$ and $p_{c}=1-(1-\tau)^{n-1}$.

It remains to specify the distribution of unit decrement time $T_{d}$. Let $T_{s}$ and $T_{c}$ be the time duration, normalized to the system slot time, of one successful and failed (collided) transmission, respectively. If CTS/RTS mechanism is used, $T_{s}$ and $T_{c}$ can be calculated as follows:

$$
\begin{aligned}
T_{s}= & \text { RTS }+ \text { CTS }+\mathrm{HDR}+L+\mathrm{ACK}+3 \times \mathrm{SIFS} \\
& +\mathrm{DIFS}, \\
T_{c}= & \text { RTS + DIFS }
\end{aligned}
$$

otherwise

$$
\begin{aligned}
& T_{s}=\mathrm{HDR}+L+\mathrm{ACK}+\mathrm{SIFS}+\mathrm{DIFS}, \\
& T_{c}=\mathrm{HDR}+L+\mathrm{DIFS} .
\end{aligned}
$$

In both cases, $L$ denotes the length of the data payload of a Wi-Fi frame. Let $p_{s}$ be the probability that one of the other $n-1 \mathrm{Wi}-\mathrm{Fi}$ station transmits successfully (note it is generally not true that $p_{s}=1-p_{c}$ ); that is,

$$
\begin{aligned}
p_{s} & =(n-1) \tau(1-\tau)^{n-2} \\
& =(n-1)\left[\left(1-p_{c}\right)^{(n-2) /(n-1)}+p_{c}-1\right] .
\end{aligned}
$$

The unit decrement time $T_{d}$ has following pmf:

$$
p_{T_{d}}\left(t_{d}\right)= \begin{cases}1-p_{c} & t_{d}=1 \\ p_{c}-p_{s} & t_{d}=T_{c} \\ p_{s} & t_{d}=T_{s} \\ 0 & \text { o.w. }\end{cases}
$$

\subsection{Formulation of Wi-Fi and Duty Cycled LTE-U Coexistence.} Consider an infrastructure-based Wi-Fi network coexisting with an LTE-U network on the same unlicensed band, where interference is coming from LTE-U subsystem to the Wi-Fi station labeled Sta-A. Considering a duty cycle period which extends T Wi-Fi system slots (refer to Figure 1) the eNB or UE in LTE-U subsystem transmits during the LTE-U ON stage of duration $\alpha T$, where $\alpha \in[0,1]$, and keeps silence during the LTE-U OFF stage (as discussed in [3], even during LTE$\mathrm{U}$ quiet period, the reference signal may have same significant interference to Wi-Fi transmission. In this paper, we assume the LTE-U being completely off during its OFF stage). The variables $T$ and $\alpha$ are defined to be LTE-U duty cycle period and duty cycle, respectively. The Wi-Fi subsystem does not cooperate with LTE-U nor has any prior knowledge about LTE- $U$ interference; it simply transmits data frame based on the DCF mechanism.

As has been introduced before, assuming only one out of the $n$ Wi-Fi stations receives LTE-U interference is for 
the purpose of maintaining the Markov properties to model the rest of $n-1$ stations, so when $n$ is large enough, the collision probability $p_{i}$ and unit decrement time $T_{d}$ can still be approximately computed using the generalized Markov chain model. The $n-1$ noninterference stations actually provide a stationary background Wi-Fi traffic for Sta-A.

We denote a Wi-Fi subsystem as $\mathscr{H}(q, L, n)$, where $q \in$ $[0,1]$ is the Wi-Fi collision probability subject to LTE-U interference (the Wi-Fi transmission failure probability, when a Wi-Fi frame and an LTE-U frame transmits simultaneously, only applies to Sta-A), $L$ is the data payload length in each transmission, and $n$ is the number of clients in the $\mathrm{Wi}-\mathrm{Fi}$ subsystem which determines the Wi-Fi to Wi-Fi collision probability (i.e., Wi-Fi collision probability in the absence of LTE-U transmission). Furthermore let $(T, \alpha)$ denote an air time sharing scheme with duty cycle period $T$ and LTE- $U$ duty cycle $\alpha$.

Instead of adopting the uniformed Wi-Fi throughput as in [11], we evaluate the Wi-Fi throughput (saturation throughput of Sta-A, the same premise keeps for future discussion) $R(T, \alpha, \mathscr{H})$ as the number of bits can be successfully transmitted per Wi-Fi slot time. The Wi-Fi service time $D(T, \alpha, \mathscr{H})$, or the medium access delay, is defined to be the time interval (also normalized to system slot time) from the time instant that a packet becomes the head of the queue and starts to contend for transmission, to the time instant that either the packet is acknowledged for a successful transmission or the packet is dropped. Note both $R(T, \alpha, \mathscr{H})$ and $D(T, \alpha, \mathscr{H})$ are random variables according to above definition. Finally, we define the average Wi-Fi throughput and average service time $\mathscr{R}(T, \alpha, \mathscr{H})$ (in terms of bits/WiFi slot time) and $\mathscr{D}(T, \alpha, \mathscr{H})$ (in terms of Wi-Fi time slots), respectively, for a coexistence system with Wi-Fi subsystem $\mathscr{H}(q, L, n)$ and air time sharing scheme $(T, \alpha)$ as

$$
\begin{aligned}
& \mathscr{R}(T, \alpha, \mathscr{H})=E[R(T, \alpha, \mathscr{H})], \\
& \mathscr{D}(T, \alpha, \mathscr{H})=E[D(T, \alpha, \mathscr{H})] .
\end{aligned}
$$

For convenience, we sometimes omit the underlying variables $(T, \alpha, \mathscr{H})$ and just use a simple notation as letter $R$ or $\mathscr{R}=E[R]$ for short.

2.3. Definition of Fairness. It is a critical task to define what fairness means in this context, since there could be many ways to describe the fairness in such a coexistence scenario. One straightforward way is to compare the Wi-Fi performance with and without the presence of LTE-U. More specifically, we want to find answer to the question: Will the performance loss (throughput degradation and service time increase) due to time sharing be proportional to the duty cycle $\alpha$ ? Also, what reference values should be used when we characterize the performance loss? The definition below gives an intuitive way of measuring fairness.

Definition 1. For a given $\mathscr{H}(q, L, n)$, assume the reference Wi-Fi performance to be $\mathscr{R}(\infty, 0, \mathscr{H})$ and $\mathscr{D}(\infty, 0, \mathscr{H})$. The throughput fairness $\phi_{R}(T, \alpha, \mathscr{H})$ is the difference between the average throughput loss ratio and the LTE-U duty cycle $\alpha$; that is,

$$
\phi_{R}(T, \alpha, \mathscr{H})=\frac{\mathscr{R}(\infty, 0, \mathscr{H})-\mathscr{R}(T, \alpha, \mathscr{H})}{\mathscr{R}(\infty, 0, \mathscr{H})}-\alpha
$$

and service time fairness $\phi_{D}(T, \alpha, \mathscr{H})$ is the difference of average service time increase ratio to $\alpha /(1-\alpha)$; that is,

$$
\phi_{D}(T, \alpha, \mathscr{H})=\frac{\mathscr{D}(T, \alpha, \mathscr{H})-\mathscr{D}(\infty, 0, \mathscr{H})}{\mathscr{D}(\infty, 0, \mathscr{H})}-\frac{\alpha}{1-\alpha} .
$$

Depending on $\mathscr{H}$ and $(T, \alpha)$, the fairness measures $\phi_{R}$ and $\phi_{D}$ can be negative, positive, or zero. If both these two parameters $\left(\phi_{R}\right.$ and $\left.\phi_{D}\right)$ are zero, Wi-Fi performs at exact $(1-\alpha)$ "portion" of the non-LTE duty cycle performance. We consider such a time sharing scheme to be acceptable and reasonable. From this perspective, we have the following definition.

Definition 2. A Wi-Fi LTE-U coexistence system with WiFi subsystem $\mathscr{H}$ and air time sharing scheme $(T, \alpha)$ is fair in throughput if $\phi_{R} \leq 0$ and fair in service time if $\phi_{D} \leq 0$. If a scheme is both fair in throughput and service time, the scheme is fair.

Remark 3. Please note $q$ is a function of LTE-U to Wi-Fi INR and Wi-Fi SNR. Formulating the collision probability $q$ is out of the scope of this paper. It is obvious that very low INR/SNR interference causes almost no impact to Wi-Fi system which is trivial; that is, $q \approx 0$ and $\phi_{R}, \phi_{D} \leq 0$. This paper focuses on the situations when INR and SNR are comparable, and in most subsequent discussions we assume $q=1$, which means a Wi-Fi transmission will definitely fail if an LTE-U transmission occurs at the same time. Additionally, we will show in Section 4 how $\phi_{D}$ and $\phi_{R}$ decay when $q$ increases from 1 to 0 in a numerical example. Readers are reminded that $q=1$ can happen to either strong or weak interference cases.

\section{Impact of Duty Cycled LTE-U Interference}

During the LTE-U ON period, the ith attempt of Wi-Fi transmission fails with probability

$$
p_{i}^{\prime}=1-\left(1-p_{c}\right)(1-q)
$$

Whether the failure probability should be chosen as $p_{i}$ or $p_{i}^{\prime}$ depends on whether the LTE-U is on or off; therefore the Sta-A DCF could no longer be modeled by Markov chain. Instead, we characterize the Sta-A throughput and service time in three steps:

(1) Suppose a Wi-Fi packet leaves Sta-A buffer at time $T_{0}=t_{0}$, where $t_{0} \in\{0,1, \ldots, T-1\}$, and at time $T_{e}$, where $T_{e} \in\left\{t_{0}, \ldots, \infty\right\}$; the packet will be either sent out successfully or dropped. Conditioning on $t_{0}$, we compute the conditional distribution $p_{T_{e} \mid T_{0}}\left(t_{e} \mid t_{0}\right)$ of the finish time $T_{e}$, the conditional mean service time $E\left[D \mid t_{0}\right]$, and conditional mean throughput $E\left[R \mid t_{0}\right]$. 
(2) Let $T_{e}^{\prime}$ be a function of $T_{e}$ that

$$
T_{e}^{\prime}=T_{e} \quad \bmod (T-1)
$$

and the conditional distribution of $T_{e}^{\prime}$ can be derived from $p_{T_{e} \mid T_{0}}\left(t_{e} \mid t_{0}\right)$ as

$p_{T_{e}^{\prime} \mid T_{0}}\left(t_{e}^{\prime} \mid t_{0}\right)=\sum_{T_{e}: T_{e} \bmod (T-1)=t_{e}}^{+\infty} p_{T_{e} \mid T_{0}}\left(t_{e} \mid t_{0}\right)$.

If we regard the $T$ time slots (labeled as $0, \ldots, T-1$ ) in a duty cycle period as $T$ states, those $T$ states form the state space of a one-dimension Markov chain, with transition probability from state $t_{0}$ to state $t_{e}^{\prime}$ of

$$
p_{T_{e}^{\prime} \mid T_{0}}\left(t_{e}^{\prime} \mid t_{0}\right)
$$

because knowing the start time $t_{0}$ and the distribution of $T_{e}^{\prime}$ does not depend on previous packet transmission start times. The distribution $p_{T_{0}}\left(t_{0}\right)$ can be computed as the stationary distribution over these $T$ states.

(3) Lastly, the Sta-A mean throughput $E[R]$ and mean service time $E[D]$ can be derived as $E[R]=$ $\sum_{t_{0}=0}^{T-1} p_{T_{0}}\left(t_{0}\right) E\left[R \mid t_{0}\right]$ and $E[D]=\sum_{t_{0}=0}^{T-1} p_{T_{0}}\left(t_{0}\right) E[D \mid$ $\left.t_{0}\right]$.

3.1. The Conditional Probability $p_{T_{e} \mid T_{0}} p\left(t_{e} \mid t_{0}\right)$ under Weak LTE- $U$ Interference. When LTE-U interference is weak, StaA keeps transmitting in the LTE-U ON stage whenever possible. For some $t_{0}$, consider an $m$ dimension vector $\mathbf{w}^{(m)}=$ $\left(w_{0}, \ldots, w_{m-1}\right) \in\left\{0, \ldots, \mathrm{CW}_{0}-1\right\} \times \cdots \times\left\{0, \ldots, \mathrm{CW}_{m-1}-1\right\}$, where $1 \leq m \leq M+1$. A vector $\mathbf{w}^{m}$ with $1 \leq m<$ $M+1$ fully determines a back-off pattern and also uniquely determines the transmission finish time $t_{e}$. When $m=M+1$, depending on the last trial being successful or not, the finish time has two possibilities. Hence the distribution $p_{T_{e} \mid T_{0}}\left(t_{e} \mid\right.$ $t_{0}$ ) can be derived by first getting the conditional distribution
$p_{\mathbf{W}^{(m)} \mid T_{0}}\left(\mathbf{w}^{(m)} \mid t_{0}\right)$, where $\mathbf{W}^{(m)}$ is the corresponding random variable to $\mathbf{w}^{(m)}$.

The time of $i$ th transmission attempt $\zeta_{i}$ is also a function of $\mathbf{w}$ that

$$
\begin{aligned}
\zeta_{i}\left(\mathbf{w}^{(m)}\right)=t_{0}+E\left[T_{d}\right]\left(\sum_{k=0}^{i} w_{k}+i T_{c}\right) & \\
& 0 \leq i \leq m-1,
\end{aligned}
$$

and comparing $\zeta_{i}$ with the values of $\alpha T, T,(1+\alpha) T, 2 T, \ldots$, it could be figured out if the $i$ th attempt falls within the LTE-U ON period, record the result by a bool function

$$
\begin{aligned}
& g\left(\zeta_{i}\right) \\
& \quad= \begin{cases}0 & \alpha T \leq \zeta_{i} \bmod T<\left(\zeta_{i}+T_{s}\right) \bmod T<T \\
1 & \text { o.w }\end{cases}
\end{aligned}
$$

and combining (11) and (16), we have

$$
\begin{aligned}
p\left(\mathbf{w}^{(m)} \mid t_{0}\right) & \\
= & \left(\prod_{i=0}^{m-2} \frac{1-\left(1-p_{c}\right)\left(1-q g\left[\zeta_{i}\left(\mathbf{w}^{(m)}\right)\right]\right)}{\mathrm{CW}_{i}}\right) \\
& \cdot\left(\frac{\left(1-p_{c}\right)\left(1-q g\left[\zeta_{m-1}\left(\mathbf{w}^{(m)}\right)\right]\right)}{\mathrm{CW}_{m-1}}\right) .
\end{aligned}
$$

The end time $T_{e}$ is a function of random vector $\mathbf{W}^{(m)}$ and $T_{e}\left(\mathbf{W}^{(m)}\right)$ is the sum of the total waiting time and the time of each transmission of back-off pattern $\mathbf{W}^{(m)}$. Let $h\left(\mathbf{W}^{(m)}\right)$ be a function of random variable $\mathbf{W}^{(m)}$ of the successful retransmission probability of the $m$ th retrial,

$$
h\left(\mathbf{W}^{(m)}\right)=\left(1-p_{c}\right)\left(1-q g\left(\zeta_{m-1}\left(\mathbf{W}^{(m)}\right)\right)\right) .
$$

According to the DCF, the mapping $f:\left(\mathbf{W}^{(m)}, t_{0}\right) \rightarrow T_{e}$ is

$$
\begin{aligned}
& f\left(\mathbf{W}^{(m)}, t_{0}\right) \\
& = \begin{cases}t_{0}+E\left[T_{d}\right] \zeta_{m-1}+T_{s} & 1 \leq m<M+1 \\
t_{0}+h\left(\mathbf{W}^{(M)}\right) E\left[T_{d}\right]\left(\sum_{i=0}^{M} W_{i}+M T_{c}+T_{s}\right)+\left(1-h\left(\mathbf{W}^{(M)}\right)\right) E\left[T_{d}\right]\left(\sum_{i=0}^{M} W_{i}+(M+1) T_{c}\right) & m=M+1 .\end{cases}
\end{aligned}
$$

Note in (19), to make $f\left(\mathbf{W}^{(m)}, t_{0}\right)$ a map, we have to deal with the map between $\left(\mathbf{W}^{(M+1)}, t_{0}\right) \rightarrow T_{e}$ so it has unique image, and we take the last retransmission duration to be its expectation. The finish time $T_{e}$ has pmf:

$$
p_{T_{e} \mid T_{0}}\left(t_{e} \mid t_{0}\right)=\sum_{\mathbf{w}: f(\mathbf{w})=t_{e}} p_{\mathbf{W} \mid T_{0}}\left(\mathbf{w} \mid t_{0}\right) .
$$

Knowing the distribution of $T_{e}$, the conditional mean $E(D \mid$ $t_{0}$ ) can be written as

$$
E\left[D \mid t_{0}\right]=E\left[T_{e}-t_{0}\right]
$$


For $E\left[R \mid t_{0}\right]$, we need to find out the probability that a packet transmission started at $t_{0}$ being dropped, denoted as $p_{\mathrm{dr}}\left(t_{0}\right)$,

$$
p_{\mathrm{dr}}\left(t_{0}\right)=\sum_{\mathbf{w}^{(M+1)}} p_{\mathbf{W} \mid T_{0}}\left(\mathbf{w}^{(M+1)} \mid t_{0}\right)\left[1-h\left(\mathbf{w}^{(M+1)}\right)\right]
$$

and the conditional mean throughput can be written as

$$
E\left[R \mid t_{0}\right]=L\left(1-p_{\mathrm{dr}}\left(t_{0}\right)\right) E\left[\frac{1}{D} \mid t_{0}\right] .
$$

3.2. The Conditional Probability $p_{T_{e} \mid T_{0}}\left(t_{e} \mid t_{0}\right)$ under Strong $L T E-U$ Interference. Under strong interference, the Wi-Fi back-off timer will be blocked when LTE-U is on. As will be demonstrated later, it effectively helps Wi-Fi to eliminate the LTE-U interference. Not only does the LTE-U interference cause the collision to $\mathrm{Wi}-\mathrm{Fi}$, but also the unit decrement time $T_{d}$ at Wi-Fi part will have a time variant pmf. When LTE-U is on, the Wi-Fi mean unit decrement time $E\left[T_{d}^{\prime}\right]$ becomes $\alpha T$ (when $\alpha T$ is chosen at a reasonable value, e.g., $T \geq 100$ ). As a result, (15) and (19) which both reply on $E\left[T_{d}\right]$ no longer hold true. Computation of $\zeta_{i}(\mathbf{w})$ and $T_{e}=f\left(\mathbf{W}^{(m)}, t_{0}\right)$ needs iterative algorithm, which is given below; this algorithm has complexity $\mathrm{O}\left(n^{2}\right)$. Shortly speaking, the counting process keeps checking if $T_{e} \bmod T<\alpha T$ in every iteration; if it is true then a constant $(\alpha T-S \bmod T)$ is added to the partial sum of $T_{e}$.

$$
\begin{aligned}
& T_{e}=t_{0} \\
& \text { for } i=0: m-1 \\
& \text { for } j=0: w_{i}-1 \\
& \text { if }\left(T_{e} \bmod T<\alpha T\right) \\
& T_{e}=T_{e}+\alpha T-T_{e} \bmod T \\
& \text { else } \\
& T_{e}=T_{e}+E\left[T_{d}\right] \\
& \text { end } \\
& T_{e}=T_{e}+T_{c} \\
& \text { elseif }(m \neq M+1) \\
& T_{e}=T_{e}+T_{s} ; \\
& \text { elseif }(m=M+1) \\
& T_{e}=T_{e}+(1-h(\mathbf{w})) T_{c}+h(\mathbf{w}) T_{s}
\end{aligned}
$$

end

\section{Fairness Evaluation: Monte Carlo Analysis}

It is computationally unpractical to characterize the distribution of $p_{D}\left(\cdot \mid t_{0}\right)$ as a function of $p\left(\mathbf{W} \mid t_{0}\right)$ in closed form, since the sample space of random vector $\mathbf{W}_{i}^{(M)}$ has cardinality of $\prod_{i=0}^{M-1} \mathrm{CW}_{i}$, which is at the scale of $10^{11}$. However, based on the analytical discussion in the previous section, it is easy to implement a Monte Carlo analysis for both the weak and strong interference cases. The essential idea of Monte Carlo analysis, also named as Monte Carlo simulation, is to use repeated random sampling to obtain numerical results and analyze problems that might be deterministic in principle. The procedure in our evaluation is stepped as follows.

(1) Let the first packet be generated at time 0 (slot time), and at the same time, LTE-U starts its first duty cycle.

(2) For each packet transmission/retransmission, which may be affected by collision (either Wi-Fi to $\mathrm{Wi}$ $\mathrm{Fi}$ or LTE-U to Wi-Fi, or both), multiple transmission trials may occur during one transmission/retransmission, and we uniformly generate a sample vector $\mathbf{w}=\left\{w_{0}, \ldots, w_{M-1}\right\}$ from its sample space $\prod_{i=0}^{M-1}\left\{0, \ldots, \mathrm{CW}_{i}-1\right\}$ and then a Boolean vector $\boldsymbol{\epsilon}=\left\{\epsilon_{0}, \ldots, \epsilon_{M-1}\right\}$ indicating success/failure for each trial whose distribution depends on (1) the value of $\mathbf{w}$ which determines if the $i$ th WiFi transmission trial, $i \in\{0, \ldots, M-1\}$, will be overlapping with active LTE-U transmission; (2) WiFi to Wi-Fi collision probability $p_{c}$; (3) LTE-U to WiFi collision probability $q$.

(3) Based on the given $\mathbf{w}$ and $\boldsymbol{\epsilon}$, we can deterministically compute the service time of the $k$ th transmission and record it.

(4) Let the next transmission start immediately (because we are evaluating saturate performance), and repeat steps (2)-(3) until we derive enough numerical results for analysis.

(5) The average service time $E[D]$ as well as the throughput $E[R]$ can be approximated using recorded service time from each transmission.

We refer to average throughput and average service time simply as throughput and service time. The impact of LTE-U duty cycle is discussed in terms of the following parameters:

(i) duty cycle period $T$;

(ii) the duty cycle $\alpha$;

(iii) LTE-U to Wi-Fi collision probability $q$;

(iv) Wi-Fi payload length $L$.

On the other hand, the parameters below are fixed:

(i) Wi-Fi system is saturated, that is, $\lambda=0$.

(ii) RTS/CTS is applied, slot time $\sigma=9 \mu \mathrm{s}$, Wi-Fi physical layer bit rate is $1 \mathrm{Mb} / \mathrm{s}$, for the random back-off, $M=$ $6, \mathrm{CW}_{0}=16$, and other parameters are with respect to IEEE 802.11n standard in the $5 \mathrm{GHz}$ band.

(iii) The scenario contains $17 \mathrm{Wi}-\mathrm{Fi}$ client stations, according to [12], and we know the $\mathrm{Wi}-\mathrm{Fi}$ to $\mathrm{Wi}-\mathrm{Fi}$ collision probability $p_{c}=0.3739$. 


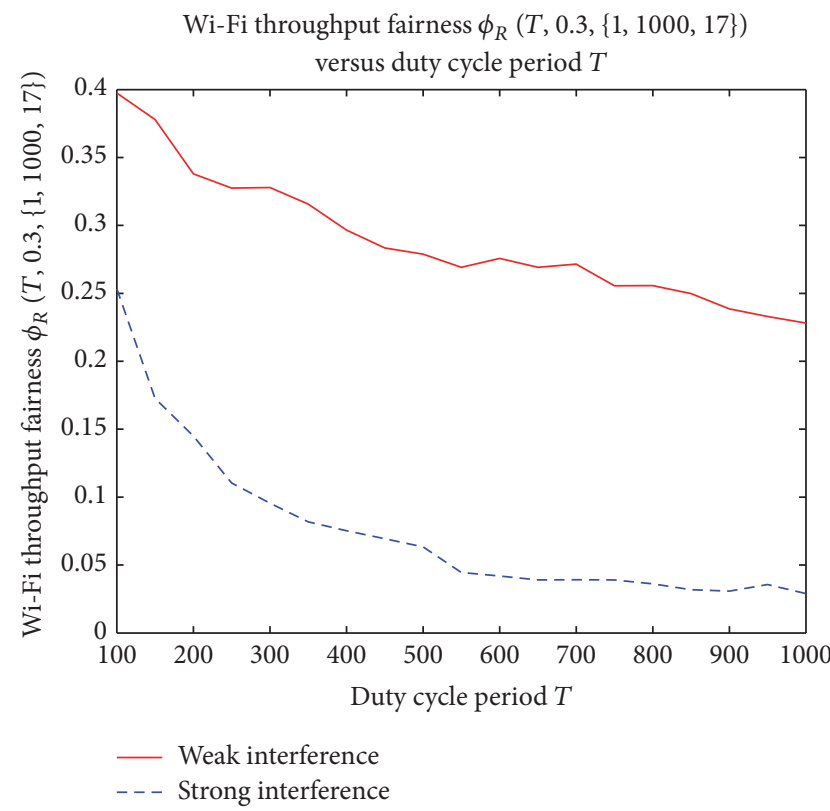

(a) Throughput fairness versus $T$
Wi-Fi service time fairness $\phi_{D}(T, 0.3,\{1,1000,17\})$

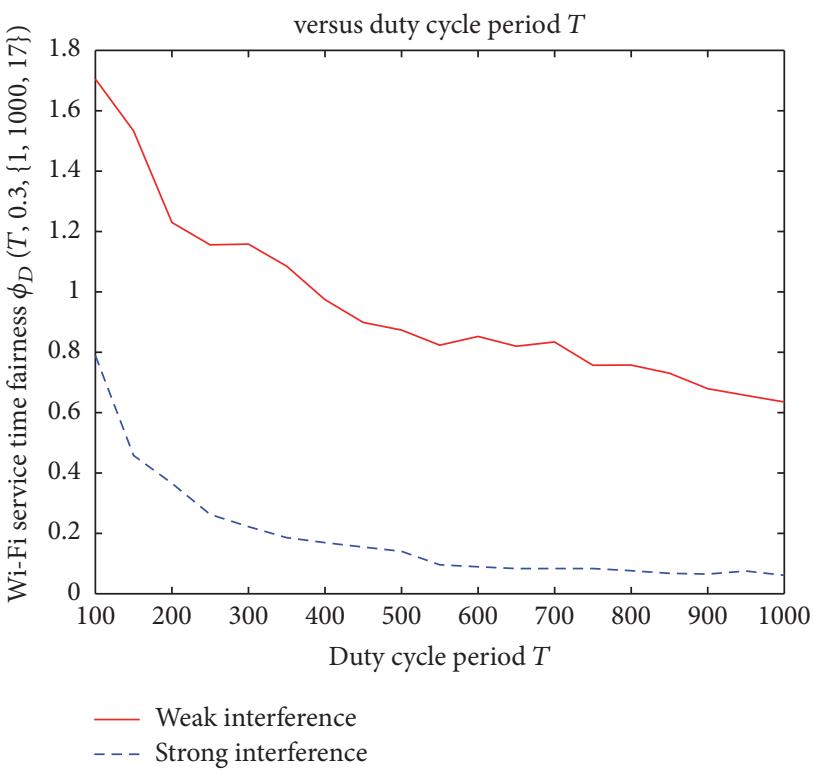

(b) Service time fairness versus $T$

FIGURE 3: Impact of duty cycle period $T$.

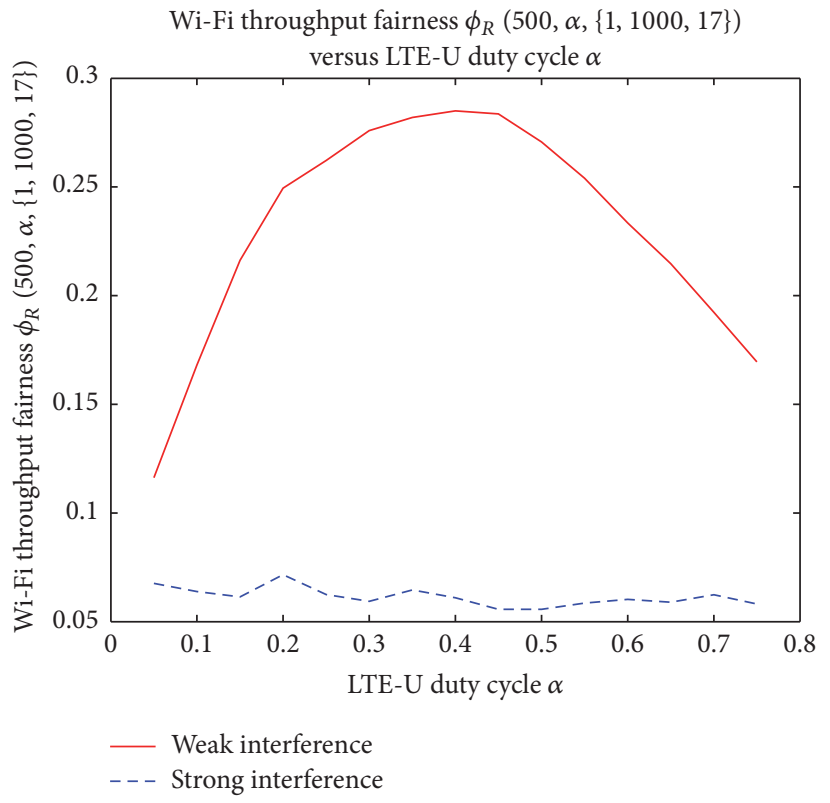

(a) Throughput fairness versus $\alpha$

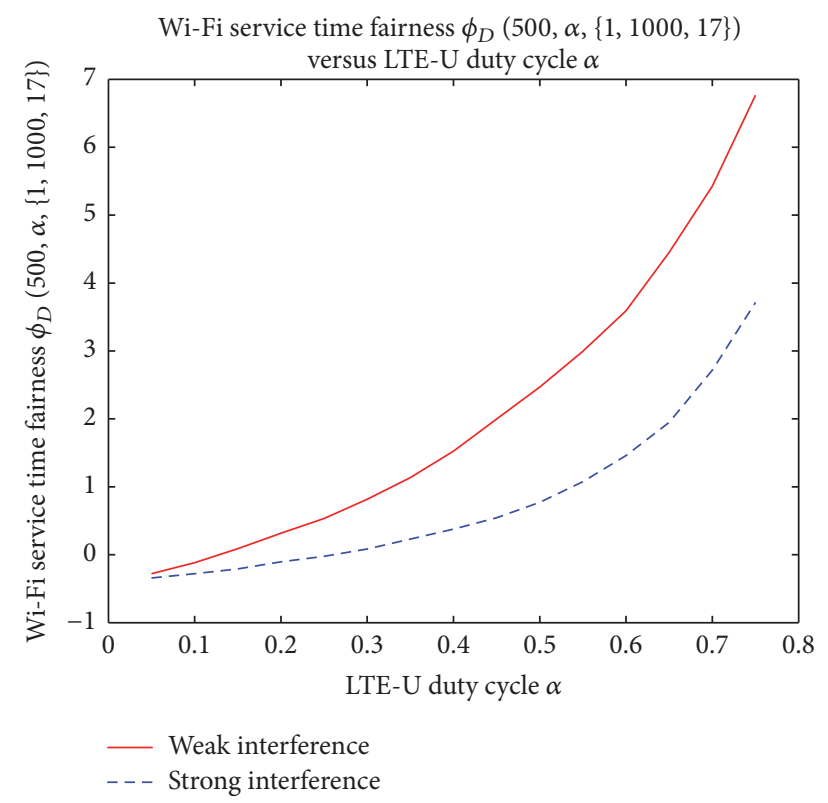

(b) Service time fairness versus $\alpha$

FIgURE 4: Impact of duty cycle $\alpha$.

4.1. The Role of Duty Cycle Period T. Fixing the duty cycle $\alpha=$ $0.3, q=1$, and $L=1 \mathrm{~KB}$, Figure 3 shows the impact of duty cycle period $T$ on throughput and service time, for both weak and strong interference scenarios. It can be observed that $T \leq$ $600 \mathrm{~ms}$ (note one LTE-U frame duration is $10 \mathrm{~ms}$ ) will cause significant $\mathrm{Wi}-\mathrm{Fi}$ performance degradation which is unfair to $\mathrm{Wi}-\mathrm{Fi}$. When $T$ is large enough, the air time sharing tends to cause less unfairness to Wi-Fi under strong interference, and more numerical results show (omitted due to the page limit) large $T$ causing less unfairness under weak interference as well.

4.2. The Role of Duty Cycle $\alpha$. For $T=500 \mathrm{~ms}, q=1$, and $L=1 \mathrm{~KB}$, the result is demonstrated in Figure 4 . When the interference is strong, throughput loss ratio is almost $\alpha$ and is linear. However, if interference is weak but signifi- 


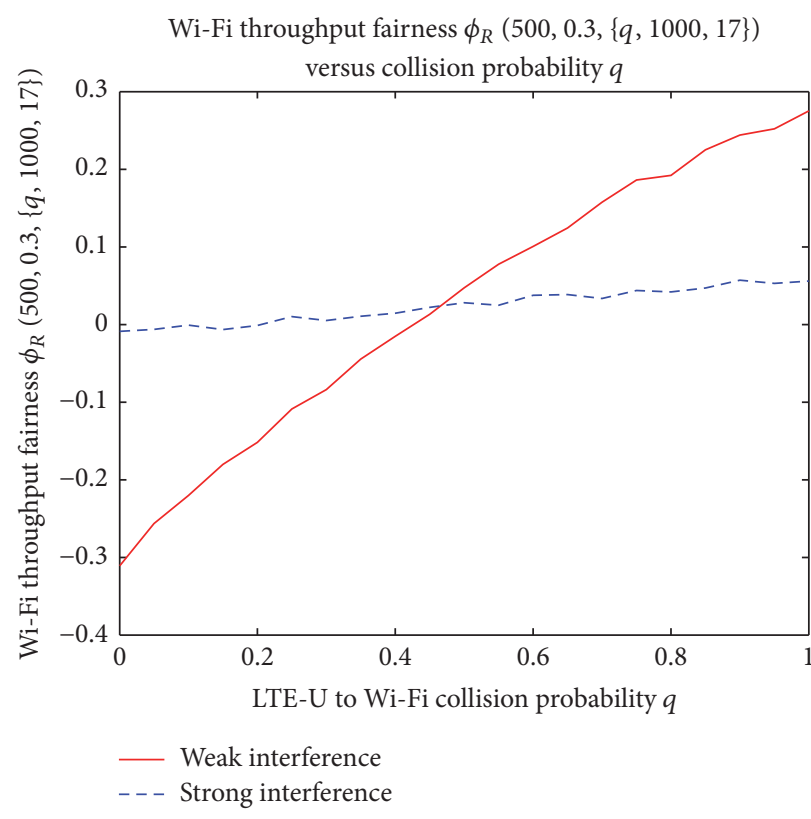

(a) Throughput fairness versus $q$

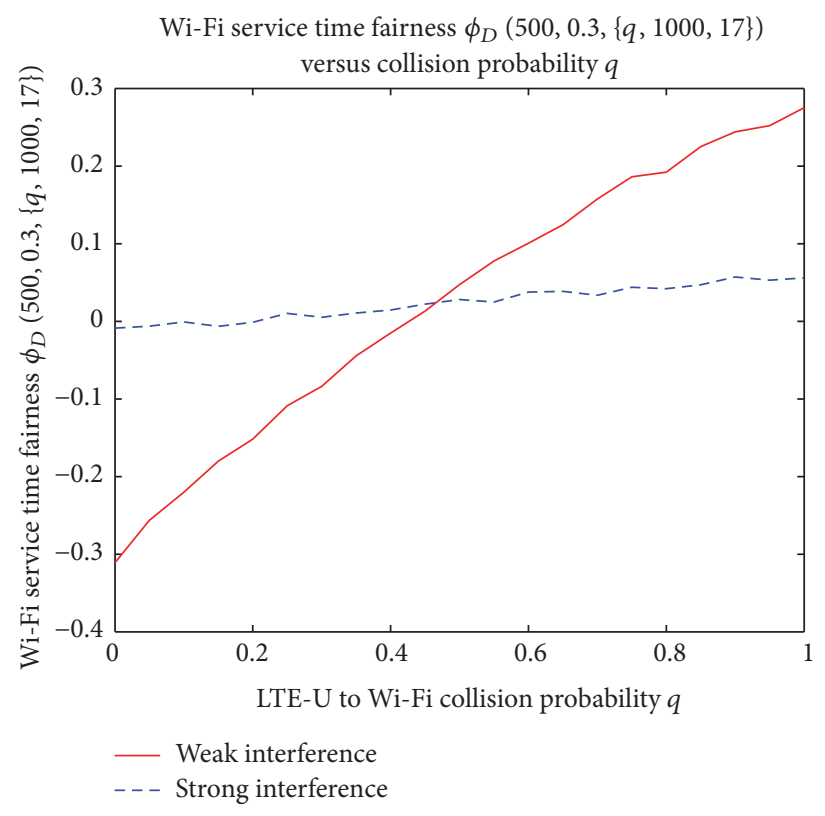

(b) Service time fairness versus $q$

FIgURE 5: The role of LTE-U to Wi-Fi collision probability $q$.

cant $(q=1)$, there can be additional reduction on throughput. When $\alpha \approx 0.4$, the air time sharing causes greatest throughput unfairness. When $\alpha \leq 0.3$, the delay seems to be linear; however, when $\alpha \rightarrow 1$, the service time increases exponentially. Recall the definition of fairness and it can be inferred for in the given network setting, a fair air time sharing scheme should have at least $\alpha \lesssim 0.3$. Considering the throughput only, strong interference approaches the throughput fairness over almost any $\alpha \in[0,1]$.

4.3. LTE-U to Wi-Fi Collision Probability q. Fixing the duty cycle $T=500 \mathrm{~ms}$ and $\alpha=0.3$ and $L=1 \mathrm{~KB}$, Figure 5 shows how the fairness varies with $q$, and it degrades almost linearly with $q$. Particularly, $q$ has less effect to fairness under strong interference because interference over $-62 \mathrm{dbm}$ will freeze the $\mathrm{Wi}$-Fi back-up timer and the only possible LTE-U to $\mathrm{Wi}$ Fi collision occurrence is when an LTE-U transmission starts after a Wi-Fi transmission.

4.4. The Role of Wi-Fi Payload Length L. Fixing $T=500 \mathrm{~ms}$, $q=1$, and $\alpha=0.3$, Figure 6 illustrates the impact of data length $L$, for both weak and strong interference, and fairness degrades almost linearly with $L$.

4.5. Why Weak Interference Is Worse at $q=1$ ? In the strong interference case, Sta-A eliminates interference by freezing the back-off timer, and after LTE-U being off, StaA could be immediately released from LTE-U interference, so the total loss ratio of the performance is very close to the duty cycle $\alpha$, as can be seen in Figure 4. However, when interference is weak, Sta-A continues to contend the channel, and it eliminates the interference by enlarging the contention window size and increasing the number of attempts during the LTE-U ON stage. Once LTE-U switches off, Sta-A will not start transmitting until the current back-off timer is reset, and in the worst case, the recovery time could take as long as

$$
2^{6} \mathrm{CW}_{0} E\left[T_{d}\right] \approx 1024 E\left[T_{d}\right] .
$$

$E\left[T_{d}\right]$ in the above experiments is about $2.6 \mathrm{~ms}$. In case $T$ is about $100 \mathrm{~ms}$, Sta-A would wait for 10 duty cycle periods long before sensing the channel again. This effect is demonstrated clearly in Figures 3 and 4.

From the information theory perspective, when LTE-U interference is strong, Wi-Fi has accurate and updated channel state information (CSI) on whether LTE-U is on or off; hence Wi-Fi could use this CSI to skip the interference. When LTE-U interference is weak, Wi-Fi has very delayed CSI since the AP knows the interference only after detecting the failure of a previous transmission, which causes significant performance degradation.

\section{Conclusion}

In this paper, we study the performance of an infrastructurebased Wi-Fi network when its operating channel in the unlicensed spectrum is air time shared with an LTE-U network. We define and characterize the Wi-Fi average performance and fairness in the presence of duty cycled LTE$\mathrm{U}$ as functions of Wi-Fi subsystem parameters and the air time sharing scheme being used. Through Monte Carlo analysis, we numerically demonstrate the fairness under typical coexistence settings. It can be observed from the results that Wi-Fi and LTE-U coexistence using simple air time sharing are generally unfair to Wi-Fi. We conclude that some other schemes (e.g., similar to the listen-before-talk mechanism used in 802.11 networks) need to be developed for LTE-U networks in order to overcome the observed unfairness. 


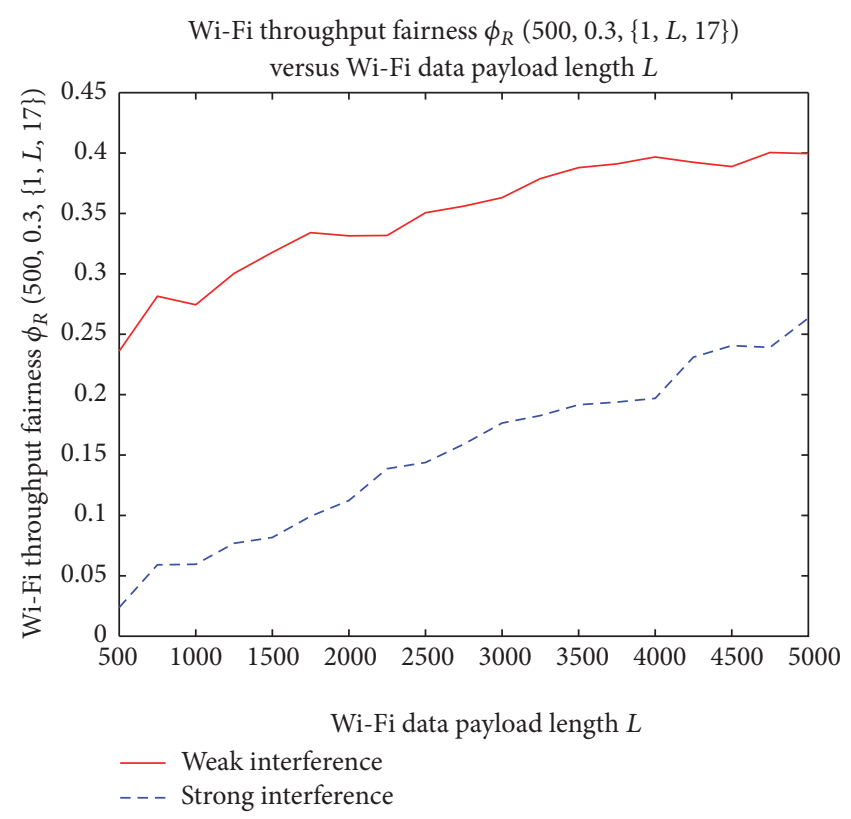

(a) Throughput fairness versus $L$

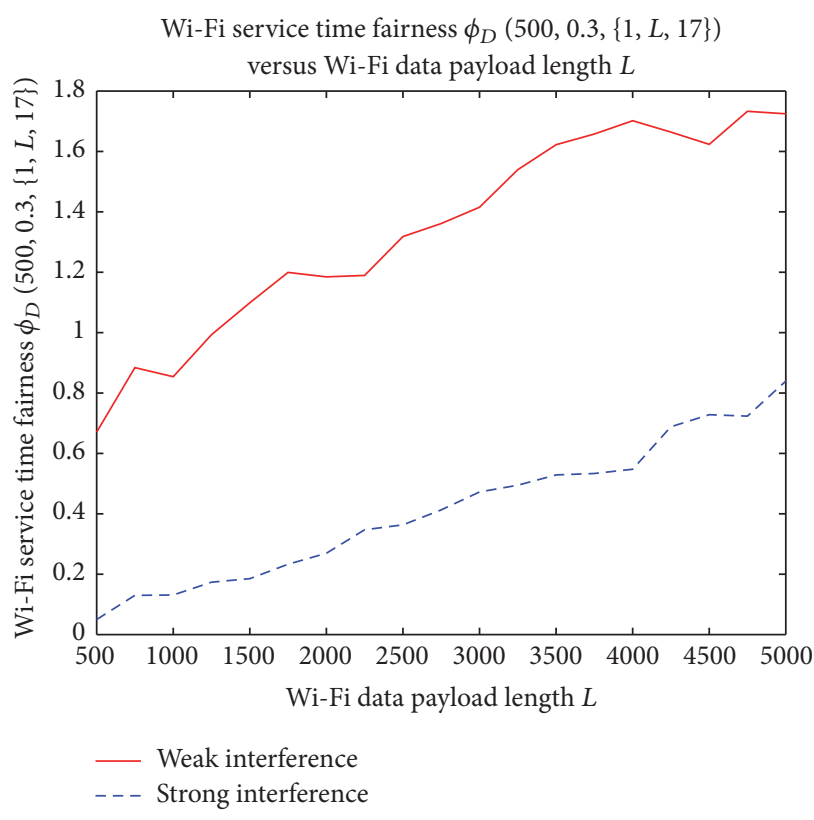

(b) Service time fairness versus $L$

Figure 6: Impact of Wi-Fi payload length $L$.

\section{Competing Interests}

The authors declare that they have no competing interests.

\section{References}

[1] "Extending the benefits of LTE-A to unlicensed spectrum," Qualcomm Whitepaper, April 2014.

[2] A. M. Cavalcante, E. Almeida, R. D. Vieira et al., "Performance evaluation of LTE and Wi-Fi coexistence in unlicensed bands," in Proceedings of the IEEE 77th Vehicular Technology Conference (VTC '13), pp. 1-6, June 2013.

[3] A. Babaei, J. Andreoli-Fang, and B. Hamzeh, "On the impact of LTE-U on Wi-Fi performance," in Proceedings of the IEEE International Symposium on Personal, Indoor and Mobile Radio Communications (PIMRC '14), Washington, DC, USA, September 2014.

[4] U-LTE: unlicensed spectrum utilization of LTE, Huawei Whitepaper, 2014.

[5] C. Cano and D. J. Leith, "Coexistence of WiFi and LTE in unlicensed bands: a proportional fair allocation scheme," in Proceedings of the IEEE International Conference on Communication Workshop (ICCW'15), pp. 2288-2293, June 2015.

[6] “LTE-U SDL Coexistence Specifications," LTE-U Forum.

[7] 3GPP TR 36.889.0.4.0, “Technical specification group radio access network; study on licensed-assisted access to unlicensed spectrum; (Release 13)".

[8] R. Ratasuk, N. Mangalvedhe, and A. Ghosh, "LTE in unlicensed spectrum using licensed-assisted access," in Proceedings of the IEEE Globecom Workshops (GC Wkshps '14), pp. 746-751, IEEE, Austin, Tex, USA, December 2014.

[9] A. M. Voicu, L. Simić, and M. Petrova, "Coexistence of picoand femto-cellular LTE-unlicensed with legacy indoor Wi-Fi deployments," in Proceedings of the IEEE International Conference on Communication Workshop (ICCW '15), pp. 2294-2300, IEEE, London, UK, June 2015.

[10] R. Kwan, R. Pazhyannur, J. Seymour et al., "Fair co-existence of Licensed Assisted Access LTE (LAA-LTE) and Wi-Fi in unlicensed spectrum," in Proceedings of the 7th Computer Science and Electronic Engineering (CEEC '15), pp. 13-18, Colchester, UK, September 2015.

[11] G. Bianchi, "Performance analysis of the IEEE 802.11 distributed coordination function," IEEE Journal on Selected Areas in Communications, vol. 18, no. 3, pp. 535-547, 2000.

[12] H. Zhai, Y. Kwon, and Y. Fang, "Performance analysis of IEEE 802.11 MAC protocols in wireless LANs," Wireless Communications and Mobile Computing, vol. 4, no. 8, pp. 917-931, 2004.

[13] A. Banchs, P. Serrano, and A. Azcorra, "End-to-end delay analysis and admission control in 802.11 DCF WLANs," Computer Communications, vol. 29, no. 7, pp. 842-854, 2006. 


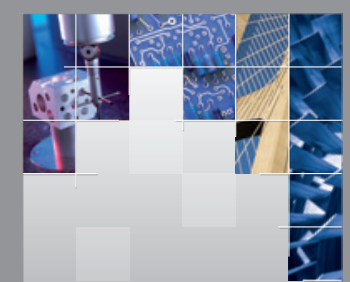

\section{Enfincering}
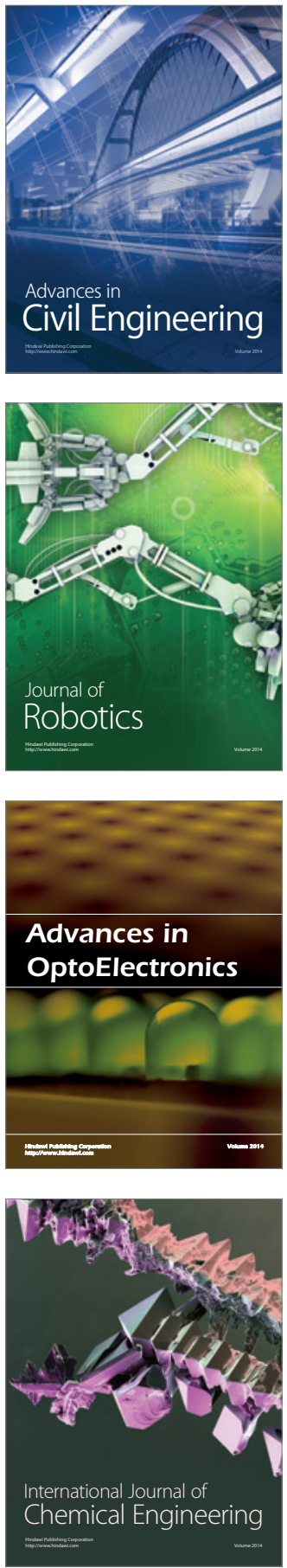

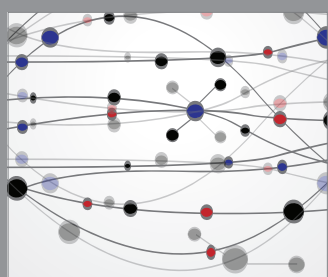

The Scientific World Journal

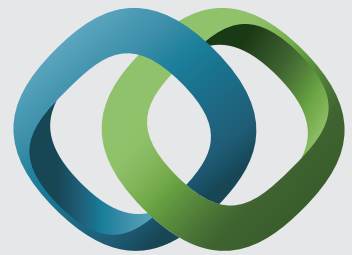

\section{Hindawi}

Submit your manuscripts at

https://www.hindawi.com
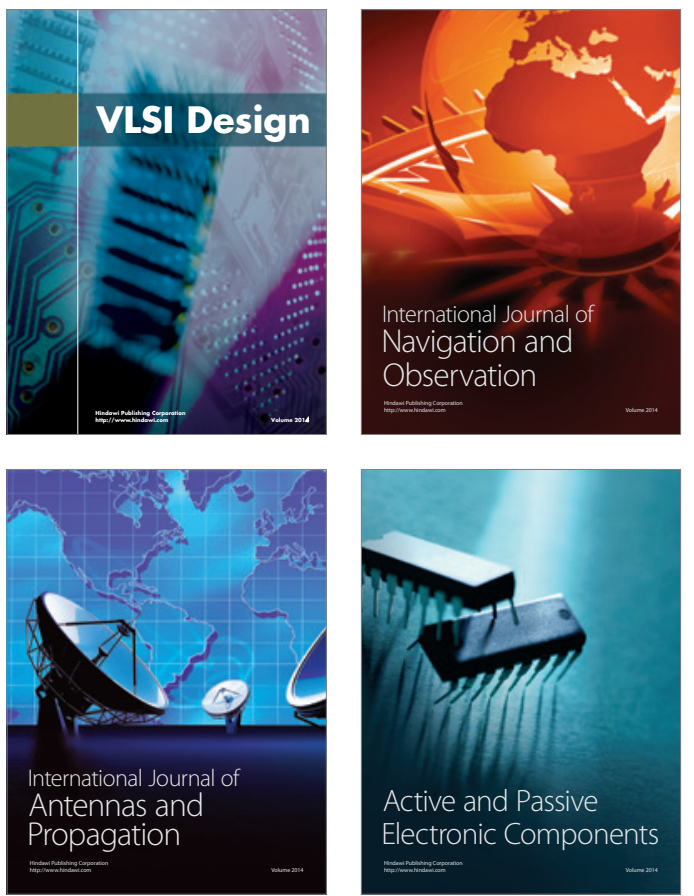
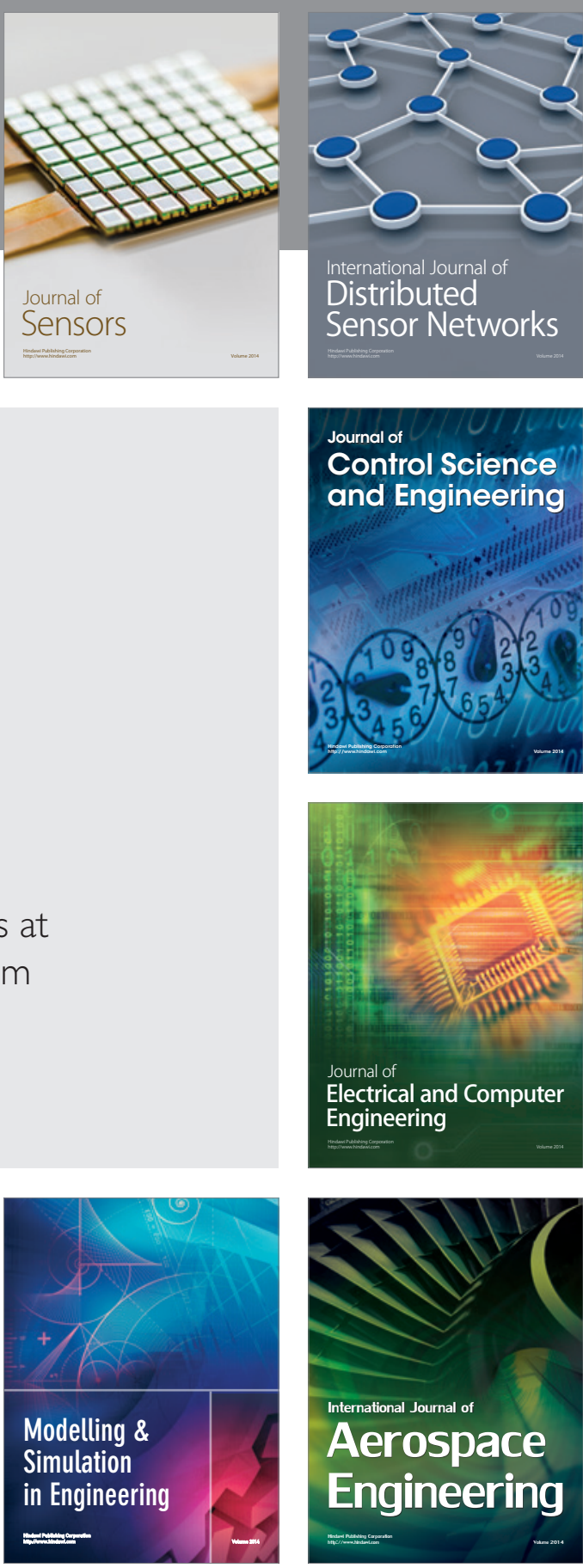

International Journal of

Distributed

Sensor Networks

$-$

Joumal of

Control Science

and Engineering
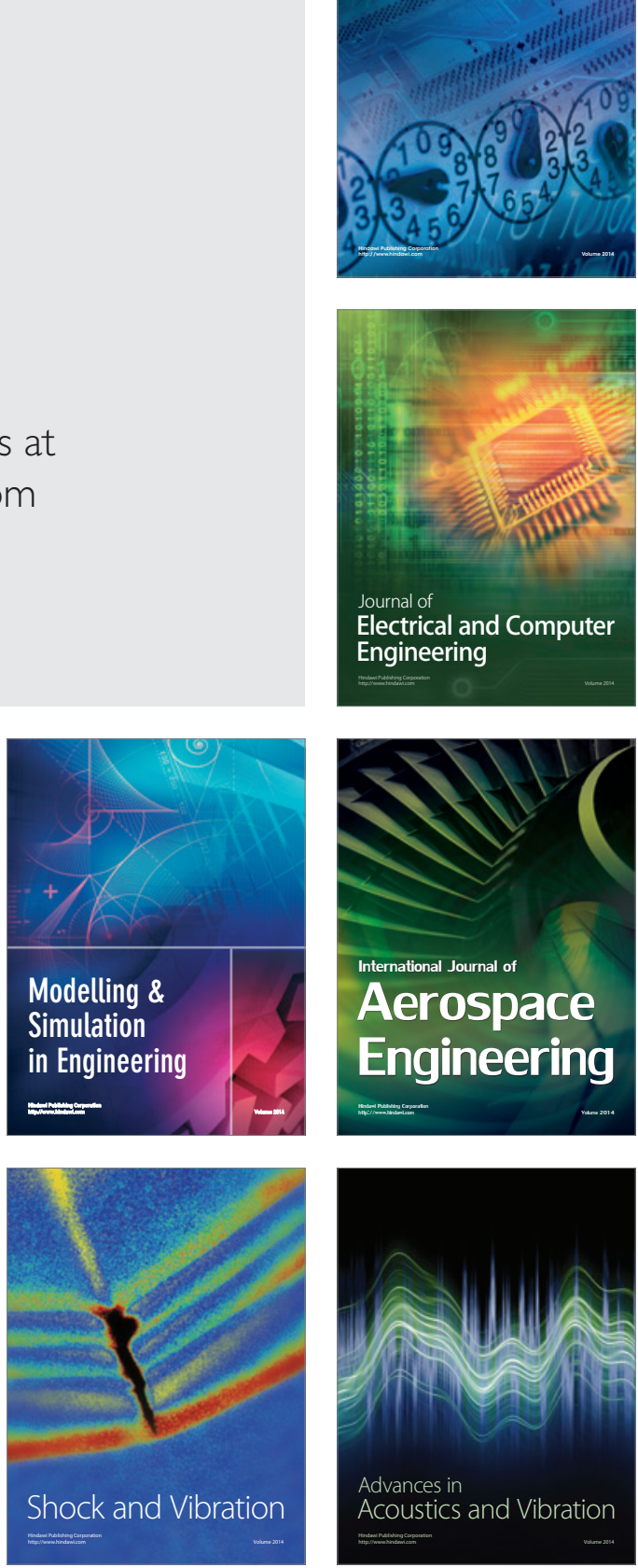